\title{
Testosteron für schwache Senioren?
}

\author{
Die Anhebung der Testosteronkonzentration im Blut von Männern über 65 hat einen \\ gering positiven Einfluss auf Sexualfunktion, Stimmung und depressive Symptome, aber \\ kaum Effekt auf die allgemeine Vitalität und die körperliche Leistungsfähigkeit.
}

\begin{abstract}
Die Testosteronkonzentration nimmt bei alternden Männern deutlich ab. Dieses Absinken wird seit langer Zeit mit dem altersbedingten Verlust an Mobilität, Sexualfunktion und allgemeiner Lebensenergie in Verbindung gebracht. Kein Wunder, dass im Zeitalter von Leistung, Fitness und Selbstoptimierung viele Männer diesem natürlichen Verlauf nicht tatenlos zusehen wollen, sondern vom Arzt hormonelle Hilfe fordern.

Ob man mit einer einzigen Substanz den altersbedingten Abbau aufhalten kann, wurde nun in einer kontrollierten Studie an 790 über 65-jährigen Männern mit einer Testosteronkonzentration von $<275 \mathrm{ng} / \mathrm{dl}$ und einer möglicherweise hypogonadal bedingten Symptomatik untersucht. Die Testosterondosis wurde dabei so gewählt, dass die Männer die
\end{abstract}

mittlere Konzentration von 19- bis 40-Jährigen aufwiesen.

Libido, erektile Funktion und sexuelle Aktivität nahmen laut den täglich geführten psychosexuellen Tagebüchern signifikant zu. Auch kam es mit der jugendlichen Testosteronkonzentration zu einer leichten Stimmungsverbesserung bzw. einer Abmilderung depressiver Symptome. In dem Teil der Studie, der die Kraft- und Ausdauerfunktion überprüfte, bestand hingegen kein Unterschied zwischen Verum und Placebo beim Anteil der Männer, die eine Zunahme um wenigstens $50 \mathrm{~m}$ beim SechsMinuten-Gehtest aufwiesen.

Kurzfristige Nebenwirkungen waren in beiden Behandlungsgruppen gleich verteilt. Bezüglich der langfristigen Risiken wollten die Forscher wegen der geringer Patientenzahl und der kurzen Dauer keine Aussage machen.

- Snyder PJ, Bhasin S, Cunningham GR et al. Effects of testosterone treatment in older men. N Engl J Med. 2016;374:611-24

\section{KOMMENTAR}

Insgesamt sind die Ergebnisse dazu angetan, die Nachfrage nach einer Testosteronsubstitution im Alter zu steigern - zumindest bei Männern, die unter der Abnahme der Sexualfunktion und dem Vitalitätsverlust leiden. Andere werden es lieber mit Plato halten, der das Greisenalter glücklich schätzte, sofern es den bis dahin uns unablässig beunruhigenden Geschlechtstrieb endlich los ist. Allerdings befanden sich die meisten Menschen zu Platos Zeiten längst nicht so lange im Greisenalter, wie das heute der Fall ist. Vielleicht würde er heute anders urteilen.

Prof. Dr. med. H. S. FüeßI

\section{Belohnende Alkoholeffekte verblassen auch bei hohem Konsum nicht}

Eine Längsschnittstudie erhellt die Rolle positiver Substanzeffekte bei der Entwicklung und Aufrechterhaltung problematischen Trinkens. 86 Probanden mit ausgeprägtem Alkoholkonsum und Binge-Drinking-Verhalten sowie 70 mit gemäßigtem Konsum wurden im Rahmen eines randomisierten, doppelblinden Studiendesigns untersucht. Sie alle waren körperlich und psychisch gesund, ihr Alter lag zwischen 21 und 35 Jahren. In zwei Testphasen zu Studienbeginn und nach fünf Jahren wurden ihnen ein alkoholhaltiges Getränk sowie ein gering alkoholhaltiges Placebo verabreicht. Danach wurden stimulierende, belohnende sowie sedierende Effekte mithilfe standardisierter Fragebögen erfasst sowie die Kortisolkonzentration im Speichel bestimmt.

Die Probanden mit hohem Alkoholkonsum zeigten bei beiden Tests eine größere Sensibilität gegenüber stimulierenden und belohnenden Alkoholeffekten. Sedierende Effekte und Kortisolkonzentrationen waren jedoch bei den Vieltrinkern geringer ausgeprägt. Je ausgeprägter das problematische Trinkverhalten war, desto deutlicher fielen die Unterschiede aus. Das zeigt, dass positiv verstärkende Mechanismen der Alkoholwirkung beim problematischen Konsum eine größere Rolle spielen als negativ verstärkende Effekte.

Die experimentellen Rahmenbedingungen, der vielleicht zu kurze Untersuchungszeitraum und die Tatsache, dass die Probanden noch keine Al- koholiker waren, schränken die Studie ein. Jedoch trägt sie zum Verständnis neuroadaptiver Vorgänge bei der Entwicklung und Aufrechterhaltung problematischen Trinkens als Vorstufe der Alkoholabhängigkeit bei.

Dr. med. C. Weinland

- King AC et al. A prospective 5-year re-examination of alcohol response in heavy drinkers progressing in alcohol use disorder. Biol Psychiatry. 2016;79:489-98

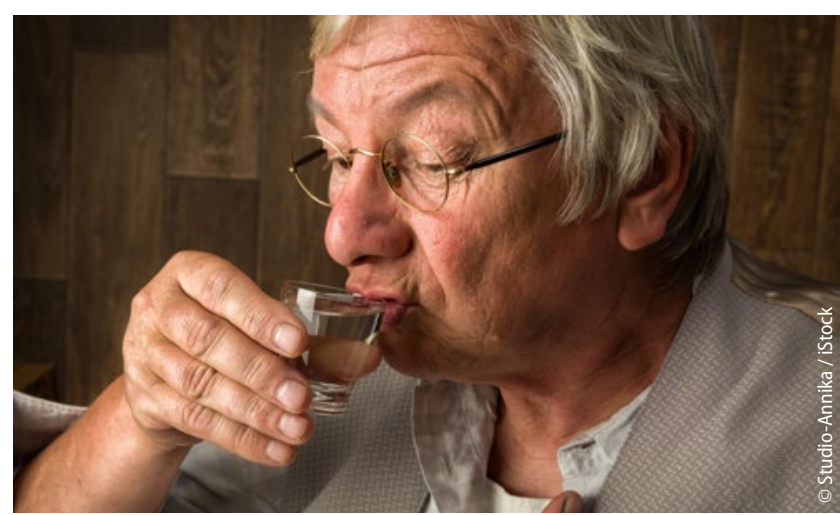

Je mehr Alkohol man trinkt, desto weniger sediert er. 